\title{
Mechanistic implications of the unique structural features and dimerization of the cytoplasmic domain of the Pseudomonas sigma regulator, PupR
}

\author{
Jaime L. Jensen ${ }^{a}$, Andrea Balbo ${ }^{b}$, David B. Neauc, Srinivas Chakravarthy ${ }^{d}$, Huaying Zhao ${ }^{e}$, \\ Sangita C. Sinhaa ${ }^{a}$, and Christopher L. Colbert ${ }^{a},{ }^{*}$ \\ aDepartment of Chemistry and Biochemistry, North Dakota State University, Fargo, ND \\ 58108-6050, USA \\ bBiomedical Engineering and Physical Science Shared Resource, National Institute of Biomedical \\ Imaging and Bioengineering, National Institutes of Health, Bethesda, Maryland, 20892, USA \\ 'Department of Chemistry and Chemical Biology, Cornell University, Northeastern Collaborative \\ Access Team, Argonne National Laboratory, Argonne, Illinois 60439, USA \\ dBio-CAT, Advanced Photon Source, 9700 South Cass Avenue, 3BIdg. 435B, Argonne, IL 60439, \\ USA \\ eDynamics of Macromolecular Assembly Section, Laboratory of Cellular Imaging and \\ Macromolecular Biophysics, National Institute of Biomedical Imaging and Bioengineering, \\ National Institutes of Health, Bethesda, Maryland, 20892, USA
}

\section{Abstract}

\begin{abstract}
Gram-negative bacteria tightly regulate intracellular levels of iron, an essential nutrient. To ensure this tight regulation, some outer membrane TonB-dependent transporters (TBDTs) that are responsible for iron import stimulate their own transcription in response to extracellular binding by an iron-laden siderophore. This process is mediated by an inner membrane sigma regulator protein (an anti-sigma factor) that transduces an unknown periplasmic signal from the TBDT to release an intracellular sigma factor from the inner membrane, which ultimately upregulates TBDT transcription. Here we use the Pseudomonas putida ferric-pseudobactin BN7/BN8 sigma regulator, PupR, as a model system to understand the molecular mechanism of this conserved class of sigma regulators. We have determined the X-ray crystal structure of the cytoplasmic antisigma domain (ASD) of PupR to 2.0 Å. Size exclusion chromatography, small angle X-ray scattering, and sedimentation velocity analytical ultracentrifugation, all indicate that in contrast to other ASDs, the PupR-ASD exists as a dimer in solution. Mutagenesis of residues at the dimer interface identified from the crystal structure disrupts dimerization and protein stability, as determined by sedimentation velocity analytical ultracentrifugation and thermal denaturation circular dichroism spectroscopy. These combined results suggest that this type of inner membrane
\end{abstract}

*To whom correspondence should be addressed: christopher.colbert@ndsu.edu. Phone: (701) 231-7946. SUPPORTING INFORMATION AVAILABLE

Sequence alignments of PupR-ASD homologs (Figure S1); SV-AUC $c(s)$ distributions for WT PupR-ASD and sedimentation data (Figure S2); ab initio bead model for PupR-ASD (Figure S3); CD spectra of WT PupR-ASD and mutants (Figure S4); thermal denaturation curves of WT PupR-ASD and mutants (Figure S5). 
sigma regulator may utilize an unusual mechanism to sequester their cognate sigma factors and prevent transcription activation.

\section{Graphical abstract}

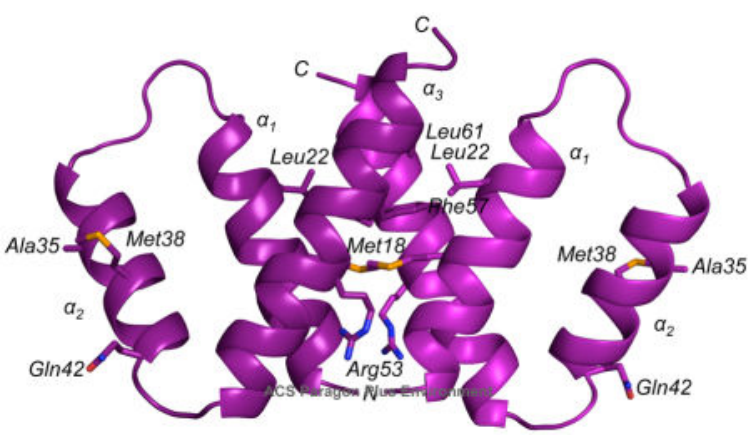

\section{Keywords}

Iron import; inner membrane sigma regulator; anti-sigma factor; X-ray crystallography; small angle X-ray scattering; analytical ultracentrifugation

Outer-membrane, cell-surface signaling networks are ubiquitous in Gram-negative bacteria $(1,2)$. The ability of these bacteria to respond to diverse environmental stimuli is dependent upon signal transduction pathways that span both the outer and inner membranes through the periplasmic space (3-5). Iron is critical for the survival of the bacterium, and in certain bacterial species, for pathogenesis. To overcome low iron bioavailability in either soil or host tissue, many bacteria secrete low molecular weight compounds called siderophores, which are high-affinity iron chelators. Well over 500 distinct siderophores have been characterized $(6,7)$. Ferrisiderophore uptake is driven by the TonB system, an energydependent protein complex located in the inner membrane $(8,9)$.

Iron levels within the cell are tightly regulated. For certain iron import systems regulation of the import proteins occurs at the transcriptional level through the extracytoplasmic function (ECF) sigma factors $(10,11)$. In these iron import systems, gene expression is initiated by association of a ligand to an outer membrane receptor protein, which triggers interactions between a periplasmic domain of the receptor protein and the $\mathrm{C}$-terminal periplasmic region of an inner transmembrane protein, interchangeably known as a sigma regulator or an antisigma factor. Activation of the sigma regulator releases an ECF sigma factor, resulting in upregulation of transcription at target promoters, such as that of a ferrisiderophore transporter $(5,12,13)$.

In the common soil bacterium Pseudomonas putida WCS358, the outer membrane pseudobactin BN7/8 transporter, PupB, signals via the inner membrane sigma regulator PupR to regulate PupI, its cognate ECF sigma factor $(14,15)$. This system is analogous to the ferric citrate uptake system (Fec) in E. coli $(11,16-18)$, the pyoverdine import system (Fpv) in Pseudomonas aeruginosa (5, 19, 20), and the heme acquisition system (Has) in Serratia marcescens (21-24). Whereas FpvR functions only as an anti-sigma factor, 
inhibiting formation of an active FpvI:RNA polymerase core (RNAP) complex (25), FecR is required for full transcriptional activation, suggesting a chaperone-like role for the FecR cytoplasmic domain (26). Similarly, in the absence of PupR, expression of PupB is significantly reduced, indicating that the anti-sigma properties of PupR potentially include both inhibition of PupI in the absence of pseudobactin, as well as activation of PupI in response to the appropriate signal $(14,15)$.

The sigma regulators, including PupR, are predicted to consist of an $\mathrm{N}$-terminal cytoplasmic region, a transmembrane helix and a C-terminal periplasmic region (25). The exact mechanism of signal transduction by the sigma regulators has not been determined, although available data suggests that controlled inner membrane proteolysis of the sigma regulator cytoplasmic domain may release the cytoplasmic sigma regulator domain and associated sigma factor $(5,27)$. In certain anti-sigma factors, such as FecR, FpvR, and FoxR, cytoplasmic or periplasmic proteolysis appears to be preceded by a conserved autoproteolytic event induced via an enzyme-independent $\mathrm{N}-\mathrm{O}$ acyl rearrangement $(13,28$, 29). However, the residues responsible for this event are not conserved in PupR. A semiconserved cytoplasmic anti-sigma domain (ASD) has been identified in multiple sigma regulators (30). The $\sigma^{70}$ superfamily, including the group IV ECF sigma factors to which PupI, FecI, and FpvI belong, is largely regulated by sigma regulators containing an ASD, despite the low sequence similarity amongst ASD-containing sigma regulators (30-32).

Release of sigma factors by structurally characterized ASDs has previously been shown to occur by two distinct mechanisms: either degradation of the ASD, or loss of affinity between the ASD and the sigma factor. The ASD of $E$. coli RseA is proteolytically degraded to release $\sigma^{\mathrm{E}}(33,34)$. The ASDs of Rhodobacter sphaeroides $\mathrm{ChrR}$ and Mycobacterium tuberculosis RslA coordinate $\mathrm{Zn}^{2+}$ and loss of the metal ion induces a loss of affinity for their cognate sigma factors $(30,35,36)$. M. tuberculosis RskA loses affinity between the ASD and the sigma factor, $\sigma^{\mathrm{K}}$, upon reduction of a disulfide bond in $\sigma^{\mathrm{K}}(37)$. However, in contrast to these sigma regulators, in FecR and FpvR $(25,26)$, and most likely also PupR $(14,15)$, the ASDs remain associated with their cognate sigma factor during transcription activation. This last group of sigma regulators has not been structurally characterized.

Here, we report the $2.0 \AA \mathrm{X}$-ray crystal structure of the cytoplasmic ASD of PupR, which unlike ASDs studied to date, has a very stable hydrophobic core and appears to be a homodimer. Biochemical characterization of the ASD in solution by small-angle X-ray scattering (SAXS), sedimentation velocity analytical ultracentrifugation (SV-AUC), and by thermal denaturation circular dichroism (CD) reveals that the dimer is a potentially important physiological state for this class of sigma regulators. This dimer provides clues to the mechanism by which these ASDs interact with their cognate sigma factors and how this class of anti-sigma factors is regulated.

\section{EXPERIMENTAL PROCEDURES}

\section{Protein Expression and Purification}

Bioinformatics analyses were used to identify residues $1-82$ as the cytoplasmic domain of Pseudomonas putida WCS358 PupR (PupR-ASD). These residues were cloned between 
NcoI and XhoI sites of the pMBP-Parallel1 vector (38). E. coli BL21(DE3)pLysS cells were transformed by this pMBP-Parallel1-PupR-ASD plasmid. Transformed cells were grown at $37^{\circ} \mathrm{C}$ in $\mathrm{LB}$ medium supplemented with $100 \mu \mathrm{g} / \mathrm{mL}$ ampicillin to an optical density $\mathrm{OD}_{600 \mathrm{~nm}}$ of $\sim 0.8$, as measured using an Implen NanoPhotometer Classic (Implen, Inc., Los Angeles, CA), and PupR-ASD expression was induced by the addition of $1 \mathrm{M}$ isopropyl thio- $\beta$-Dgalactoside (IPTG) to a final concentration of $0.5 \mathrm{mM}$. After induction, the culture temperature was maintained at $20^{\circ} \mathrm{C}$ for $18 \mathrm{hrs}$, and cells were harvested by centrifugation at $4000 \mathrm{x} \mathrm{g}$ for $30 \mathrm{~min}$. For protein purification, $30 \mathrm{~g}$ of cells were suspended in lysis buffer (25 mM HEPES pH 7.5, $150 \mathrm{mM} \mathrm{NaCl}$ ) and then lysed. The crude extract was clarified by centrifugation at $20,000 \mathrm{x}$ g for $45 \mathrm{~min}$.

At each stage of purification, protein purity was evaluated by SDS-PAGE stained with Coomassie Blue (39) and protein concentration determined by absorbance at $280 \mathrm{~nm}$ using the molar extinction coefficient $\varepsilon_{280}=16500 \mathrm{M}^{-1} \mathrm{~cm}^{-1}$ and a theoretical molecular weight of $9502 \mathrm{~g} / \mathrm{mol}$. The PupR-ASD was purified by amylose affinity chromatography followed by on-column cleavage with tobacco etch virus (TEV) protease. The supernatant was loaded onto an amylose affinity column and washed with lysis buffer plus $2 \mathrm{mM}$ DTT. TEV protease was added to the column in a 1:10 mass ratio, and incubated for $16 \mathrm{~h}$ at $4^{\circ} \mathrm{C}$ to remove the MBP tag. The identity of the TEV protease cleavage product was confirmed by ESI mass spectrometry to be consistent with an 86-residue product, containing an Nterminal, 4-residue cloning artifact (GAMG) followed by PupR residues 1-82. PupR-ASD was eluted from the column by the addition of wash buffer. The protein was loaded onto a MonoQ anion exchange column equilibrated with $25 \mathrm{mM}$ Tris $\mathrm{HCl}, \mathrm{pH}$ 7.8. Contaminants after amylose affinity bound to the MonoQ column, but the PupR-ASD did not and eluted in the wash fractions. Fractions containing the PupR-ASD were pooled, concentrated, and loaded onto tandem HR 10/30 Superdex 200-Superdex 75 size exclusion columns. Isocratic elution with $25 \mathrm{mM}$ HEPES $\mathrm{pH} 7.5,150 \mathrm{mM} \mathrm{NaCl}$ at a flow rate of $0.4 \mathrm{~mL} / \mathrm{min}$ was performed. Fractions containing PupR-ASD were pooled and concentrated to $15 \mathrm{mg} / \mathrm{mL}$ and placed at $4{ }^{\circ} \mathrm{C}$ or flash-frozen as pellets in $\mathrm{LN}_{2}$ and stored at $-80^{\circ} \mathrm{C}$ until use. This protein was estimated to be $>90 \%$ pure by Coomassie Blue stained SDS-PAGE.

Selenomethionine (SeMet)-derivatized PupR-ASD was prepared by a modified protocol involving methionine synthesis suppression $(40,41)$. Briefly, E. coli BL21(DE3)pLysS cells transformed with pMBP-Parallel1-PupR-ASD were grown at $37^{\circ} \mathrm{C}$ to saturation in $3 \mathrm{~mL} \mathrm{LB}$ media with $100 \mu \mathrm{g} / \mathrm{mL}$ ampicillin, then transferred to pre-warmed M9 minimal media containing $2 \mathrm{mM} \mathrm{MgSO}_{4}, 0.1 \mathrm{mM} \mathrm{CaCl}_{2}, 0.4 \%$ w/v glucose, and $100 \mu \mathrm{g} / \mathrm{mL}$ ampicillin and incubated at $37^{\circ} \mathrm{C}$. Once the cell density increased to an $\mathrm{OD}_{600 \mathrm{~nm}}$ of 1.0 , the media were supplemented with SeMet, Lys, Thr, Phe, Leu, Ile, and Val and the temperature lowered to $20^{\circ} \mathrm{C}$. Expression was induced by addition of $0.5 \mathrm{mM}$ IPTG, and the cultures grown for an additional $18 \mathrm{~h}$. Purification of SeMet PupR-ASD was performed as described for the native protein. Incorporation of SeMet was confirmed by ESI mass spectrometry.

\section{Preparation of Site-Directed Mutants of PupR-ASD}

Aspartate encoding point mutations of WT PupR-ASD were created using the QuikChange II protocol (Stratagene), the pMBP-Parallel1-PupR-ASD vector as a template, and primers 
containing the desired aspartate mutation, using either of the two most common codons. The mutant gene sequences were verified by DNA sequencing and the plasmids used to transform E. coli Arctic Express (DE3) competent cells. The mutant proteins were prepared in the same manner as for the WT, with the $\mathrm{pH}$ of buffers maintained at 8.0 throughout purification.

\section{Protein Crystallization}

Reproducible needle-like clusters were obtained by sitting-drop vapor diffusion by combining $1 \mu \mathrm{L}$ protein solution ( $12 \mathrm{mg} / \mathrm{mL}$ PupR-ASD in $25 \mathrm{mM}$ HEPES $\mathrm{pH} 7.5,150 \mathrm{mM}$ $\mathrm{NaCl}$ ) with $1 \mu \mathrm{L}$ reservoir solution (100mM Bis-Tris $\mathrm{pH} 6.0$, and $3 \mathrm{M} \mathrm{NaCl})$ and equilibrating against $500 \mu \mathrm{L}$ reservoir solution at $20^{\circ} \mathrm{C}$. These crystals were used for microseeding fresh drops by serial dilution and resulting in the growth of larger crystal clusters. These larger clusters were broken into $\sim 200 \mu \mathrm{m}$ x $20 \mu \mathrm{m}$ x $20 \mu \mathrm{m}$ single crystals, and cryo-protected by passing through MiTegen LV CryoOil (MiTegen, Ithaca, NY) prior to flash-freezing in liquid nitrogen. Crystals of SeMet PupR-ASD were grown and harvested in a similar manner.

\section{Diffraction Measurements and X-ray Structure Determination}

Diffraction data were collected at NE-CAT beamlines 24ID-C and 24ID-E at the Advanced Photon Source (APS), Argonne National Laboratory, Argonne, IL. All data sets were processed using the RAPD automated processing suite (https://rapd.nec.aps.anl.gov/rapd), which incorporates XDS for integration and scaling (42). The SeMet PupR-ASD structure was determined by single-wavelength anomalous dispersion phasing (SAD) using intensities measured at $12.66 \mathrm{keV}$. Three of the five selenium sites (four in the PupR-ASD sequence, and one from the N-terminal cloning artifacts) were located, and initial phasing performed with AutoSol in PHENIX (43). These phases were used to calculate initial electron density maps for automated model building with AutoBuild (44). Refinement was carried out in PHENIX (45) and iterative manual model building in COOT (46). The SeMet PupR-ASD structure was subsequently used as a molecular replacement (MR) model to phase a $2.0 \AA$ data set of native PupR-ASD (47). MR was performed using Phaser-MR (48), followed by Autobuild (44) and refinement in PHENIX, using automated TLS group determination in PHENIX (49). Water oxygen atoms were positioned using PHENIX, with subsequent visual verification. Data collection and refinement statistics are summarized in Table 1. All figures were prepared using PyMOL v.1.5.0.4 (Schrodinger). Analysis of surface areas, protein interfaces, assemblies, and interactions were determined using the PISA server (http:// www.ebi.ac.uk/pdbe/pisa) (50). RMSD comparisons were carried out in PyMOL. Atomic models and structure factors have been deposited into the Protein Data Bank under PDB entry $5 \mathrm{CAM}$ and $5 \mathrm{COS}$.

\section{Size Exclusion Chromatography Small-Angle X-ray Scattering (SEC-SAXS) Data Collection and Analysis}

SEC-SAXS was performed on the WT PupR-ASD at Bio-CAT beamline 18ID of the APS. An inline Superdex 75 10/300 column was equilibrated with $25 \mathrm{mM}$ Tris $\mathrm{pH} 7.8,150 \mathrm{mM}$ $\mathrm{NaCl}$ at a flow rate of $0.7 \mathrm{~mL} / \mathrm{min}$. SEC-SAXS data were recorded at a wavelength of 1.033 
$\AA$ on a Pilatus $31 \mathrm{M}$ detector at a sample to detector distance of $3.0 \mathrm{~m}$, covering a momentum transfer range of $0.0055 \AA^{-1}<q<0.3316 \AA^{-1}$. Data were normalized to the incident X-ray beam intensity, and buffer subtracted prior to analysis using the ATSAS program suite (51). Linearity of the Guinier region was examined using PRIMUS (52) and the Kratky plot calculated to assess disorder. $\operatorname{GNOM}(53,54)$ was used to compute the radius of gyration, $R_{g}$; pairwise distribution function, $P(r)$; and the maximum particle dimension, $D_{\max }(53,54)$. Ten independent $a b$ initio bead model reconstructions were calculated using DAMMIF (55) by enforcing P2 symmetry. The resultant dummy atom models were averaged and filtered with DAMAVER (56) with default parameters. The models had an average $\chi^{2}$ of $1.20 \pm 0.014$, with an averaged normalized spatial discrepancy value for the ten DAMMIF (55) calculations of $0.853 \pm 0.189$. The resultant threedimensional averaged envelope was superimposed upon the PupR-ASD crystal structure using SITUS and the UCSF Chimera package (57-59). CRYSOL (60) was used to compare theoretical scattering curves calculated for monomer and dimer atomic models against the experimental SAXS data. PyMOL v.1.5.0.4 was used to visually evaluate fits of the PupRASD and for manual docking. The molecular weight of the PupR-ASD was determined using the SAXS MoW server (http://www.if.sc.usp.br/ saxs/) (61). Theoretical scattering profiles calculated for the PupR-ASD monomer and dimer X-ray structures were fit to the experimental scattering profiles using the FoxS server (http:// www.modbase.compbio.ucsf.edu/foxs/) $(62,63)$. EOM $2.0(64,65)$ in the ATSAS suite was employed to model the flexible N- and C-termini of the PupR-ASD. The full Pup-ASD sequence, along with either the monomer dimer crystal structure, was used to generate 10,000 random conformers, which were subsequently pooled by EOM to generate ensembles that best fit the experimental data. The theoretical scattering profiles of the best models, as identified by the program, were fit to the experimental scattering intensities with the FoxS server.

\section{Analytical Ultracentrifugation}

Sedimentation velocity analytical ultracentrifugation (SV-AUC) experiments were performed in a Beckman ProteomLab XL-I analytical ultracentrifuge (Beckman Coulter, Indianapolis, IN) at $20^{\circ} \mathrm{C}$ following the standard protocol (66) and boundary profile analysis (67). PupR-ASD samples in a buffer of $25 \mathrm{mM}$ HEPES pH $8.0,150 \mathrm{mM} \mathrm{NaCl}$ were loaded into a sample cell assembly containing a standard double-sector charcoal-filled epon centerpiece with a $12 \mathrm{~mm}$ or $3 \mathrm{~mm}$ pathlength and sapphire windows. Sample cell assemblies loaded in a rotor were equilibrated at $20^{\circ} \mathrm{C}$ for at least $2 \mathrm{hrs}$ prior to sedimentation. Sedimentation profiles were acquired using absorbance optics at $280 \mathrm{~nm}$ in combination with interference optics at $20^{\circ} \mathrm{C}$ at 201,240 $\mathrm{g}$ (rotor speed of 50,000 rpm at a radius of $7.2 \mathrm{~cm})$.

Various concentrations were explored for each protein construct to populate monomer and dimer species. For WT PupR-ASD, seven samples were prepared with concentrations that ranged from $1.1 \mu \mathrm{M}-102 \mu \mathrm{M}$. Mutant PupR-ASD proteins were prepared at a few concentrations in this range. The monomer-dimer self-association of each sample was characterized using previously reported procedures (66). Briefly, a standard $c(s)$ model (67) was used to analyze the sedimentation profiles. Precise concentrations were determined 
using refractive index increments, $d n / d c$, predicted from the amino acid sequence of PupRASD in SEDFIT (68) and subsequent integration of the $c(s)$ peaks from the interference data, resulting in the total protein concentration in $\mathrm{mg} / \mathrm{mL}$ corresponding to the integrated peaks in the $c(s)$ distributions. Signal weighted-average $s$-value $\left(s_{w}\right)$ isotherms (69) were created by integration of the $c(s)$ peaks in the distribution between 0 and $4 \mathrm{~S}$ for the data and plotted as a function of protein concentration in SEDPHAT (69). A monomer-dimer selfassociation model was applied to the $s_{w}$ isotherms in SEDPHAT (70). For WT PupR-ASD, $\mathrm{s}$-value of the monomer was fixed at $1.06 \mathrm{~S}$, based on the value determined from the mutants, which do not form dimers under the concentration range tested; $s$-value of the dimer was fitted along with the binding constant $\left(K_{\mathrm{d}}\right)$. For the mutants, $s$-value of monomer and dimer were fixed at $1.06 \mathrm{~S}$ and $1.71 \mathrm{~S}$ (determined from the WT $s_{\mathrm{W}}$ isotherm) respectively; thus, the only fitted parameter is the $K_{\mathrm{d}}$. All experimental SV data and best-fit values are presented in units of experimental $s$-values. Error analysis of $K_{\mathrm{d}}$ for WT was performed using the error surface projection method and F-statistics (71) and the 95\% confidence intervals were reported.

\section{Circular Dichroism (CD) Spectroscopy and Thermal Denaturation}

The PupR-ASD was diluted to $10 \mu \mathrm{M}(0.095 \mathrm{mg} / \mathrm{ml})$ in $10 \mathrm{mM}$ potassium phosphate $\mathrm{pH} 8.0$, $100 \mathrm{mM}\left(\mathrm{NH}_{4}\right)_{2} \mathrm{SO}_{4}$. CD spectra were measured at $4^{\circ} \mathrm{C}$ between 180 and $250 \mathrm{~nm}$ using a Jasco J-815 spectrometer with a PFD-425S Peltier cell holder and a $1 \mathrm{~mm}$ quartz cell. The spectra were buffer subtracted, and the secondary structure content estimated using K2D2 (72); as well as CONTIN, CDSSTR, and SELCON, provided in CDPro (73). CD melting curves were recorded at $222 \mathrm{~nm}$ with $10 \mu \mathrm{M}$ WT or mutant protein in CD buffer by increasing the temperature from $4^{\circ}-85^{\circ} \mathrm{C}$ in $1^{\circ} \mathrm{C}$ increments with a slope of $1^{\circ} \mathrm{C} / \mathrm{min}$. Protein unfolding was monitored during both heating and cooling. Complete $\mathrm{CD}$ spectra at $4^{\circ} \mathrm{C}$ were recorded just prior to each experiment. Melting temperatures were determined by fitting a standard Boltzmann sigmoidal curve to the ellipticity in Origin 8 (OriginLab Corp., Northampton, MA). The final melting temperature was defined as the inflection point after fitting.

\section{RESULTS}

\section{Tertiary Structure of the PupR-ASD}

The PupR-ASD crystals belonged to space group $P 2{ }_{1}$ with unit cell parameters of $a=33.5$ $\AA$ A. $b=113.8 \AA, c=34.9 \AA$ and $\beta=113.7^{\circ}$ (Table 1). Crystals of native PupR-ASD and the SeMet derivative diffracted to 2.0 and $2.2 \AA$, respectively. SeMet SAD data were used to locate 12 of the possible 20 selenium sites, enabling the placement of four monomers per crystal asymmetric unit (Figure 1). This provided a $2.2 \AA$ model of PupR-ASD. A monomer from this structure was used as a MR search model to determine the structure from the native data set at $2.0 \AA$ resolution. $\mathrm{R}_{\mathrm{work}}$ converged to $17.8 \%$ and $\mathrm{R}_{\text {free }}$ to $21.7 \%$. No Ramachandran outliers are present in the structure (Table 1).

The final model is comprised of residues 10-65 of chain A, 11-64 of chain B, 8-66 of chain $\mathrm{C}$, and 5-65 of chain D, but electron density corresponding to the $\mathrm{N}$ - and $\mathrm{C}$-termini of each monomer was not resolved. The structure of the PupR-ASD monomer is a tightly-packed, 
three-helix bundle (Figure 1). The three-helix core is stabilized by hydrophobic interactions, primarily between helices 2 and 3, including $\pi-\pi$ stacking of W40 and H47 (plane-plane distance $=4.0 \AA$ ) (Figure 2). Residues analogous to W40, W20 and W51 are completely conserved in homologous putative iron transport anti-sigma regulator proteins across bacterial species (Figure S1).

Despite sharing only 7-16\% identity (31), the PupR-ASD and other known ASDs share a common structural fold comprising a three-helix core bundle (Figure 3). PupR-ASD helices 1-3 superimpose (Figure 3) with a root-mean-square deviations (rmsd) of 2.52-3.18 $\AA$ over 40-48 residues of the ChrR-ASD from $R$. sphaeroides ((30); PDB ID 2Z2S), RseA from $E$. coli ((34); PDB ID 1OR7), RskA from M. tuberculosis ((37); PDB ID 4NQW), and RslA from M. tuberculosis ((36); PDB ID 3HUG). ChrR and RslA are both zinc-binding antisigma (ZAS) factors, with their tertiary structure stabilized by a $\mathrm{Zn}^{2+}$, which is coordinated by a conserved His- $\mathrm{X}_{3}-\mathrm{Cys}-\mathrm{X}_{2}$-Cys motif. Loss of the $\mathrm{Zn}^{2+}$ results in loss of structure and anti-sigma function. In contrast, the PupR-ASD, RseA-ASD, and RskA-ASD comprise another group of ASDs that are stabilized by hydrophobic cores, although the PupR-ASD is the only ASD of known structure that stabilizes its hydrophobic core via $\pi-\pi$ stacking of aromatic residue side chains, two tryptophans and one histidine (Figure 2). The four previously determined ASD structures also include a 4-10 residue loop preceding a fourth, C-terminal helix, whose location varies amongst the various ASDs (Figure 3). Interestingly, PupR residues 68-82, which are analogous to this region, are unstructured in the PupR-ASD structure.

\section{PupR-ASD Forms a Dimer in Solution}

The relative molecular weight and oligomeric state of the native PupR-ASD was determined by analytical size exclusion chromatography during purification. The relative molecular weight of PupR-ASD subunit as estimated by SDS-PAGE is approximately $9000 \mathrm{Da}$, consistent with the expected molecular weight of $9502 \mathrm{Da}$ for this domain. However, the PupR-ASD eluted from the size exclusion column as a single Gaussian-shaped peak with an apparent molecular mass of $19 \mathrm{kDa}$, suggesting that the PupR-ASD purified as a homodimer.

Small-angle X-ray scattering in conjunction with size-exclusion chromatography (SECSAXS) was performed on the purified PupR-ASD to characterize its solution state (Figure 4a). The Guiner plot was linear in the $0<q<0.1 \AA^{2}$ range (Figure $4 \mathrm{~b}$ ), verifying that the sample was monodisperse. The Guinier plot indicated PupR-ASD has an $R_{g}=18.69 \pm 0.02$ $\AA$ (Figure $4 \mathrm{~b}$ ), with the $\mathrm{P}(\mathrm{r})$ analysis yielding a $D_{\text {max }}$ corresponding to $64.5 \AA$ (Figure $4 \mathrm{c}$ ). The $R_{g}$ is 1.4 -times the $R_{g}$ calculated from the crystal structure of a single PupR-ASD monomer. The Kratky plot analysis (Figure 4d) revealed that the PupR-ASD is partially unfolded, consistent with the X-ray structure, wherein the $11 \mathrm{~N}$-terminal and $18 \mathrm{C}$-terminal residues are not observed in the electron density map, and therefore presumed to be disordered. An estimate for the PupR-ASD molecular weight was calculated from the scattering data with the maximum $q$ set to $0.15 \AA^{-1}$. This calculation indicated the species in solution had a molecular weight of $19.8 \mathrm{kDa}$, demonstrating that the dimer was the dominant 
species in the SEC-SAXS sample. Thus, the SEC and the SAXS results are consistent with, and indicative of, the PupR-ASD existing as a homodimer in solution.

SV-AUC was utilized to quantify PupR-ASD self-association. Sedimentation coefficient distributions $-c(s)$ of WT- were obtained for SV data at seven different concentrations, ranging from $1.1 \mu \mathrm{M}$ to $101.9 \mu \mathrm{M}$, of WT PupR-ASD. The monomer had a sedimentation coefficient of $\sim 1.06 \mathrm{~S}$, while the dimer had a sedimentation coefficient of $\sim 1.71 \mathrm{~S}$ (Figure $\mathrm{S} 2)$. An $s_{w}$ isotherm was then calculated from the dilution series and fit with a monomerdimer equilibrium binding model (Figure 5), to obtain a $K_{d}=10.2 \mu \mathrm{M}$ (95\% confidence interval $[7.58,13.79] \mu \mathrm{M}$ ) for the WT PupR-ASD (Table 2). Interestingly, the SEC and SEC-SAXS samples were loaded at a range of concentrations from $100 \mu \mathrm{M}(1.8 \mathrm{mg} / \mathrm{ml})-$ $1.5 \mathrm{mM}(28 \mathrm{mg} / \mathrm{ml})$, i.e. at concentrations where formation of the dimer was favored. Thus, the combined SEC, SEC-SAXS and SV-AUC experiments indicate that an equilibrium exists between the monomeric and dimeric states of the ASD, and suggest that at normal physiological concentrations at the bacterial inner membrane, the ASD may transition between these states.

\section{The PupR-ASD Dimer Structure}

The X-ray crystal structure was analyzed for potential dimer interfaces. Two distinct sets of interactions were seen between different monomers in the asymmetric unit. One set consisted of asymmetric interactions stabilized by hydrogen bonding between the $\mathrm{N}$ terminus of chain $\mathrm{C}$ and the loop between helix 1 and helix 2 of chain $\mathrm{D}$ (Figure 1). The other set consisted of symmetric interactions between chain A and chain B, with substantial stabilization by hydrophobic interactions between residues on helices 1 and 3 of each monomer (Figure 1). The potential symmetric dimer created by the latter set was related by two-fold, rotational, non-crystallographic symmetry. In contrast, a closed dimer is not created by the asymmetric interactions, and it is unclear why such interactions would create only a dimer, rather than a linear chain of subunits. This suggests that the symmetric dimer observed in the crystal structure is more likely to correspond to the dimer found in solution.

We used the PISA server (50), to analyze the two potential interfaces identified in the crystal structure. For the symmetric dimer the total surface area buried upon the association of two subunits is $1740 \AA^{2}$, with a complexation significance score of 1.0. This complexation significance score assesses the maximal fraction of the total free energy of binding for a given interface, and a score of 1.0 denotes a clear significance of this interface for dimer assembly. Thus, this interaction would bury approximately $33 \%$ of the total surface area of each subunit upon formation of a symmetrical dimer. In contrast, the asymmetric interaction buries only $1395 \AA^{2}$ of total surface area, with a complexation significance score of 0.0. This provides additional support that the symmetric homodimer is likely the assembly seen in solution (Figure 4).

The symmetric homodimer is stabilized by hydrophobic packing between helices 1 and 3 of each monomer (Figure 5). Overall, helix 1 of monomer A (Helix $1^{\mathrm{A}}$ - chain id in superscript) runs parallel to helix 3 in monomer B $\left(\operatorname{Helix}^{\mathrm{B}}\right)$. This interface is stabilized by a "knobs-ingrooves" hydrophobic packing between residues M18 and L22 on Helix $1^{\mathrm{A}}$ and residues R53 (the aliphatic region), F57, and L61 on Helix $3^{\mathrm{B}}$ (Figure 5). This unit has an approximately 
$45^{\circ}$ tilt to the equivalent Helix $1^{\mathrm{B}}$ :Helix $3^{\mathrm{A}}$ unit to create a four-helix bundle. The bundle is further stabilized by interactions between the F57 and L61 of Helix ${ }^{\mathrm{A}}$ with F57 and L61 of Helix $3^{\mathrm{B}}$ (Figure 5). The asymmetric interaction involves an association between monomers $\mathrm{C}$ and $\mathrm{D}$ (Figure 1). In contrast to the symmetric interaction, the interface is a three-helix bundle consisting of Helix $1^{\mathrm{C}}, \mathrm{Helix} 3^{\mathrm{C}}$, and Helix $2^{\mathrm{D}}$. This interaction involves $\mathrm{A} 14^{\mathrm{D}}, \mathrm{M} 18^{\mathrm{D}}$, and $\mathrm{L} 22^{\mathrm{D}}$ packing against $\mathrm{L} 34^{\mathrm{C}}$ and $\mathrm{M} 38^{\mathrm{C}}$, and the aliphatic part of $\mathrm{R} 41^{\mathrm{C}}$. Additionally, there is hydrogen bonding between the main chain of residues $\mathrm{T} 7^{\mathrm{D}}, \mathrm{I} 9^{\mathrm{D}}$, and $\mathrm{G} 11^{\mathrm{D}}$ and residues $\mathrm{P} 27^{\mathrm{C}}, \mathrm{V} 29^{\mathrm{C}}$, and $\mathrm{A} 31^{\mathrm{C}}$, similar to an anti-parallel beta sheet.

Full-length PupR consists of an N-terminal cytoplasmic domain, a transmembrane domain and a C-terminal periplasmic domain. In the symmetric homodimer, the C-termini of the two monomers are positioned within 11.2 ̊̊ of each other on the same face of dimer (Figure 5), suggesting that this side faces the inner membrane, facilitating the simultaneous insertion of the two transmembrane domains from a homodimer into the inner membrane. In contrast, the hypothetical dimer based on the asymmetric contacts would position the two C-termini $41.8 \AA$ apart on opposite faces, which in the full-length protein would complicate the simultaneous insertion of the transmembrane helices into the inner membrane since the chain would run in opposite directions. Thus, we conclude that the symmetrical interactions represent the homodimer seen in solution, while the asymmetric interactions merely represent crystal contacts. Further, this symmetric homodimer likely represents a physiologically relevant state of PupR.

We analyzed the PupR-ASD SAXS data to further explore the ASD dimerization. Theoretical SAXS curves were calculated for the crystal structures of the ASD monomer and the symmetric homodimer, and these curves were fit to the experimental PupR-ASD scattering profile (Figure 4a). The experimental data fits the calculated scattering curve for the homodimer $(\chi=8.4)$ much better than that for the monomer $(\chi=23.3)$. Since both the $X-$ ray structure and the Kratky plot indicated that the ASD was partially disordered, fitting of the theoretical scattering curves of the symmetric dimer and the monomer were then done using the program EOM 2.0(64, 65). The inclusion of the disordered regions of the PupRASD in the models dramatically improved the fits of the calculated scattering curve for the homodimer (best $\chi=2.2$ ) relative to that for the monomer (best $\chi=13.5$ ). This clearly indicates that the scattering curve calculated from a model comprising of the symmetric PupR-ASD homodimer as well as the disordered regions fits best to the experimental scattering data.

A low-resolution molecular bead model was also calculated from the experimental SAXS data. The initial model showed an envelope for the PupR-ASD that was C2 symmetric in nature. Manual fitting of the symmetric dimer found in the crystal structure into the averaged ab initio SAXS envelope further supported this as the plausible assembly. An improved bead model was calculated by enforcing two-fold rotational symmetry (Figure S3). The symmetric homodimer was docked into this SAXS envelope using SITUS, with a resulting correlation coefficient of 0.87 , indicating a reasonable fit to the calculated ab initio bead model (Figure S3). Thus, the symmetric PupR-ASD homodimer represents the structure of this domain at high concentrations in solution. 


\section{Residues at the Dimer Interface are Important for ASD Stability}

In order to investigate the importance of the residues at the subunit interface within the symmetric homodimer, we decided to assess the impact of mutating these residues on the quaternary, tertiary and secondary structure of the ASD. Amongst the residues M18, L22, F57, R53, and L61 (Figure 5), found at the PupR-ASD dimer interface, the three residues with the greatest buried surface area (BSA) were presumed to be of critical importance and were selected for mutagenesis to aspartic acid to force repulsion at the interface. The three residues selected were M18 (BSA $\left.=103 \AA^{2}\right)$, L22 (BSA $\left.=120 \AA^{2}\right)$, and F57 (BSA = 109 $\AA^{2}$ ) (Figure 5). In addition to single mutants of each of these residues, we also created an M18D and L22D double mutant, and a triple Asp mutant. As a control, several other residues, involved in the asymmetric interactions that were deemed to be crystal contacts, were also mutated to aspartic acid. These include A35, M38, and Q42 (Figure 5). Lastly, we also mutated R53 (BSA $=92 \AA^{2}$ ) and L61 (BSA $=88 \AA^{2}$ ), two residues that participate in both the dimer interface and the asymmetric crystal contacts (Figure 5).

We analyzed the mutant proteins by SV-AUC to assess whether dimerization was altered compared to WT. As expected, we found that the symmetric dimer interface mutations dramatically impacted quaternary structure. Mutation of M18, L22, or F57 to Asp substantially weakened the dimer, resulting in an increase of the dimer dissociation constant to over $200 \mu \mathrm{M}$ (Table 2). Even more strikingly, mutation of R53 or L61 resulted in $K_{d}$ s of > $500 \mu \mathrm{M}$ (Table 2). In contrast, consistent with our expectations, mutation of A35, M38, and Q42, residues that are involved only in asymmetric contacts, only marginally impacted $K_{d}$ 's (Table 2). Thus, mutation of any of the residues involved in the symmetric dimer interface resulted in significant disruption of the PupR-ASD homodimer, while mutation of residues at the asymmetric interface did not have a similar effect.

Next, we employed CD spectroscopy (Figure S4) in conjunction with thermal denaturation (Figure S5) to assess how these mutations impact secondary structure and the stability of the tertiary structure. We found that wild-type (WT) PupR-ASD has a $T_{m}$ of $53.3^{\circ} \mathrm{C}$. Relative to the WT, mutation of the three residues that contribute only to the symmetric dimer interface also significantly destabilized the ASD tertiary structure (Table 2). The L22D and F57D mutations decreased the $T_{m}$ by $10^{\circ} \mathrm{C}$ and $\sim 5^{\circ} \mathrm{C}$, respectively. while the $\mathrm{M} 18 \mathrm{D}$ mutation had the largest destabilizing effect, reducing the $T_{m}$ by $16^{\circ} \mathrm{C}$ (Table 2). Unfortunately, the double and triple mutants did not maintain the ASD secondary structure and were largely unfolded. Interestingly, the R53D and L61D mutations, which had such a dramatic impact on dimerization, had a lesser influence on tertiary structure (Table 2), with the $T_{m}$ decreasing by $4.0^{\circ}-9.6^{\circ} \mathrm{C}$ (Figure S5); which was similar to the change observed for the A35D, M38D, and Q42D mutants, which are present at the asymmetric contacts.

Lastly, some of the symmetric homodimer interface mutants also disrupted secondary structure content of the PupR-ASD (Figure S4). Even more strikingly, the secondary structure of a double mutant (M18D + L22D) was almost entirely disrupted, while the secondary structure of a triple mutant (M18D + L22D + F57D) was completely disrupted. In complete contrast to these results, the secondary structure of the PupR-ASD was not disrupted by mutations of either the residues present at the asymmetric crystals contacts, 
A35D, M38D, or Q42D, or the residues involved in both sets of contacts, R53D and L61D (Figure S4). Thus, all the symmetric dimer interface mutations negatively impact dimer stability, but only some of them reduce secondary and tertiary structure stability. Further, this combined SV-AUC and CD analysis indicates that M18 is critical for maintaining all levels of ASD structure.

\section{DISCUSSION}

The PupR-ASD core fold comprises a three-helix bundle, similar to the fold found in other recently determined ASD structures. Unlike the ZAS ASDs, ChrR and RslA, the PupR-ASD has a relatively extensive hydrophobic core stabilized by W40, H47, W20, and W51. This hydrophobic stabilization is more extensive than that found in the ASDs of RseA and RskA. This stability may be of physiological importance to the function of the iron transport antisigma regulators, PupR, FecR, and FpvR, since it has been shown that these ASDs remain attached to their cognate sigma factor after activation of the sigma factor, and are required for sigma factor interaction with RNAP $(3,20,25,74)$. In contrast, ChrR and RslA are degraded and release the sigma factor during activation. Therefore, the stability of the ASD, and its independence from external stabilizing elements, such as a metal ion, is likely central to the mechanism of regulation by the iron-transport ASDs.

The fourth helix found in the ASDs of ChrR, RseA, RskA, and RslA serves to block the interaction of the sigma factor with the RNAP core. Removal of this fourth helix from the sigma factor then becomes an important step for activation of these sigma factors. The lack of an ordered fourth helix in the structure of PupR-ASD may indicate that this class of ASD regulates its sigma factor solely by tethering it to the membrane. Since the fourth helix is Cterminal to the core helical bundle of the ASD, a model wherein the sigma factor is sandwiched between the larger ASD dimer and the inner membrane would limit access of RNAP or DNA to the sigma factor. Recent evidence shows that intramembrane proteolysis of FpvR, and presumably subsequent release from the membrane, results in activation of PvdS and FpvI (27). Currently, however, we cannot rule out the possibility that the fourth helix in the PupR-ASD is ordered only in the presence of its sigma factor, PupI. In this scenario, the fourth helix might block RNAP binding until proteolysis of the ASD:sigma factor complex from the transmembrane region, upon which it would revert to a disordered state and allow association to the DNA promoter. Further studies are required to resolve these different hypotheses.

Isolation of the PupR-ASD as a dimer was unexpected. Our identification of a symmetric dimer from the X-ray crystal structure necessitated an in-depth biophysical investigation of the role of the residues at dimer interface. These investigations subsequently confirmed that the residues at this interface are critical components of dimer packing and stability. The presence of the dimer does not preclude interaction with a sigma factor. The dimeric interface does not interfere with regions of the ASD responsible for recognizing the $\sigma_{2}$ region of the sigma factor, as found in the ChrR and RseA structures (30, 34). Superposition of the ChrR: $\sigma^{\mathrm{E}}$ complex and the RseA: $\sigma^{\mathrm{E}}$ complex upon the PupR-ASD homodimer reveals that the surface analogous to the surface of these sigma regulators, which interacts with the $\sigma_{2}$ region of $\sigma^{\mathrm{E}}\left(\sigma_{2}{ }^{\mathrm{E}}\right)$, is localized to the exposed face of the PupR-ASD dimer. The C- 
terminal $\sigma_{4}$ region of $\sigma^{\mathrm{E}}\left(\sigma_{4}{ }^{\mathrm{E}}\right)$ does sterically clash with the second monomer of the PupRASD homodimer. However the flexible linker between $\sigma_{4}{ }^{\mathrm{E}}$ and $\sigma_{2}{ }^{\mathrm{E}}$ should enable the adjustments that would allow interaction of the $\sigma_{4}$ region with the second PupR-ASD subunit of the dimer. Alternatively, the $\sigma_{4}$ region may adopt an unknown alternate conformation that does not interact with the ASD allowing interaction of two sigma factors with the dimer. Comparison of the $\sigma_{4}{ }^{\mathrm{E}}$ structure from the ChrR and RseA complexes (30, 34) with that observed in the complex of $\sigma_{4}{ }^{\mathrm{E}}$ with AsiA (75) demonstrates the flexibility of the $\sigma_{4}$ region. In either case, the ASD dimer would allow binding of the sigma factor in the orientation discussed previously.

Dimerization has at least two potential mechanistic implications. First, the dimer is important prior to association with the sigma factor, but is disrupted concomitant with sigma factor association, allowing each monomer to interact with a separate sigma factor. Thus, the dimer may stabilize the ASD and protect against degradation, thereby preventing loss of sigma factor inhibition and unintended activation. However, given that some of our mutant proteins remained folded even when dimer assembly is disrupted, in this mechanism it is unclear why a dimer is necessary prior to interacting with the sigma factor.

The second mechanistic possibility is that since the ASD tethers the sigma factor to the inner membrane, the PupR-ASD dimer is necessary for tethering a dimeric state of PupI.

Currently, it is unknown whether PupI and its homologs exist as dimers. This might also explain the differential proteolysis observed in the periplasm. Draper and coworkers showed that the sigma regulator, FpvR, is degraded in the periplasm in the absence of signal, but in the presence of ferrisiderophore on the TBDT the site of proteolysis shifts to a location in the inner membrane resulting in release of the sigma factor from the membrane (27). This would require continuous synthesis of the sigma regulator and sigma factor to maintain adequate levels of inactive signaling complex until activation. Presumably, this continuous degradation / synthesis cycle would be energetically unfavorable for the cell. However, the dimer could help explain the periplasmic degradation. The dimer would be more efficient in searching the outer membrane for the signaling domain from a TBDT. Once a TBDT signaling domain is bound, the attached periplasmic domain would become resistant to proteolysis, while the unattached periplasmic domain from the second sigma regulator in the dimer would be degraded yielding only one signaling complex. However, cleavage within the inner membrane of both transmembrane helices would need to be coupled, perhaps due to close proximity or autocatalysis $(13,27,28)$. In such a mechanism, the dimeric state would not significantly delay the release of the sigma factor:ASD complex. How this class of sigma regulator proteins fully conveys the ECF to activate transcription of the TBDT iron transport genes remains under investigation.

\section{Supplementary Material}

Refer to Web version on PubMed Central for supplementary material.

\section{Acknowledgments}

The authors would like to thank Drs. Angel Ugrinov and Ganesh Bala of the NDSU Materials Characterization Laboratory for their assistance with local X-ray diffraction and mass spectroscopy experiments. The content is 
solely the responsibility of the authors and does not necessarily reflect the official views of the NIGMS, NINDS, or the NIH.

FUNDING

This work was funded by the following grants to CLC: NIH NIGMS 1R15 GM113227, NIH NCRR 2P20 RR015566, and a pilot project grant from NIH NIGMS P30 GM103332; and to SS: NIH NINDS 1R03 NS090939 and NSF MCB-1413525. JJ is supported by an NSF ND EPSCoR INSPIRE DDA \#FAR0025216. This work is incorporates research conducted at the NE-CAT beamlines, which are funded by the NIH NIGMS P41 GM103403. The Pilatus 6M detector on 24-ID-C beam line is funded by a NIH-ORIP HEI S10 RR029205. Work performed at Bio-CAT was supported by NIH NIGMS 9P41 GM103622. Use of the Pilatus $31 \mathrm{M}$ detector was provided by NIGMS 1S10OD018090-01. This research used resources of the APS, a U.S. DOE Office of Science User Facility operated for the DOE Office of Science by Argonne National Laboratory under Contract No. DE-

AC02-06CH11357. Additionally, this research utilized resources at the Intramural Research Program of the National Institute of Biomedical Imaging and Bioengineering, NIH.

\section{ABBREVIATIONS}

TBDT

ASD

ECF

Fpv

Fec

Has

RNAP

SV-AUC

CD

SEC-SAXS

IPTG

SDS-PAGE

TEV

DTT

MBP

ESI

SeMet

SAD

MR

RMSD

$\mathbf{R}_{\mathbf{g}}$

$\mathbf{P}(\mathbf{r})$

$D_{\max }$

\section{Ton-B dependent transporter}

anti-sigma domain

extracytoplasmic function

pyoverdine import system

ferric citrate uptake system

heme acquisition system

RNA polymerase

sedimentation velocity analytical ultracentrifugation

circular dichroism spectroscopy

size exclusion chromatography small-angle $\mathrm{X}$-ray scattering

isopropyl thio- $\beta$-D-galactoside

sodium dodecyl sulfate polyacrylamide gel electrophoresis

tobacco etch virus

dithiolthreitol

maltose binding protein

electrospray ionization

selenomethionine

single-wavelength anomalous dispersion

molecular replacement

root-mean-square deviation

radius of gyration

pairwise distribution function

maximum particle distribution 


$\begin{array}{ll}\boldsymbol{s}_{\boldsymbol{w}} & \text { signal weighted-average } s \text {-value } \\ \boldsymbol{K}_{\boldsymbol{d}} & \text { binding or dissociation constant } \\ \text { ZASD } & \text { zinc-binding anti-sigma domain } \\ \text { BSA } & \text { buried surface area } \\ \text { WT } & \text { wild-type }\end{array}$

\section{References}

1. Crosa JH. Signal transduction and transcriptional and posttranscriptional control of iron-regulated genes in bacteria. Microbiology and molecular biology reviews : MMBR. 1997; 61:319-336. [PubMed: 9293185]

2. Braun V, Mahren S. Transmembrane transcriptional control (surface signalling) of the Escherichia coli Fec type. FEMS Microbiol Rev. 2005; 29:673-684. [PubMed: 16102597]

3. Braun V, Mahren S, Sauter A. Gene regulation by transmembrane signaling. Biometals : an international journal on the role of metal ions in biology, biochemistry, and medicine. 2005; 18:507-517.

4. Moeck GS, Coulton JW, Postle K. Cell envelope signaling in Escherichia coli. Ligand binding to the ferrichrome-iron receptor fhua promotes interaction with the energy-transducing protein TonB. The Journal of biological chemistry. 1997; 272:28391-28397. [PubMed: 9353297]

5. Llamas MA, Imperi F, Visca P, Lamont IL. Cell-surface signaling in Pseudomonas: stress responses, iron transport, and pathogenicity. FEMS Microbiol Rev. 2014; 38:569-597. [PubMed: 24923658]

6. Drechsel, H.; Winkelmann, G. Iron Chelation and siderophores. In: Winkelmann, G.; Carrano, CJ., editors. Transition Metals in Microbial Metabolism. Harwood Academic; Amsterdam: 1997. p. $1-49$.

7. Wandersman C, Delepelaire P. Bacterial iron sources: from siderophores to hemophores. Annual review of microbiology. 2004; 58:611-647.

8. Ferguson AD, Amezcua CA, Halabi NM, Chelliah Y, Rosen MK, Ranganathan R, Deisenhofer J. Signal transduction pathway of TonB-dependent transporters. Proceedings of the National Academy of Sciences. 2007; 104:513-518.

9. Noinaj N, Guillier M, Barnard TJ, Buchanan SK. TonB-Dependent Transporters: Regulation, Structure, and Function. Annual Review of Microbiology. 2010; 64:43-60.

10. Reznikoff WS, Siegele DA, Cowing DW, Gross CA. The regulation of transcription initiation in bacteria. Annu Rev Genet. 1985; 19:355-387. [PubMed: 3936407]

11. Brooks BE, Buchanan SK. Signaling mechanisms for activation of extracytoplasmic function (ECF) sigma factors. Biochimica et Biophysica Acta (BBA) - Biomembranes. 2008; 1778:19301945. [PubMed: 17673165]

12. Paget MS. Bacterial Sigma Factors and Anti-Sigma Factors: Structure, Function and Distribution. Biomolecules. 2015; 5:1245-1265. [PubMed: 26131973]

13. Bastiaansen KC, Ibanez A, Ramos JL, Bitter W, Llamas MA. The Prc and RseP proteases control bacterial cell-surface signalling activity. Environmental microbiology. 2014; 16:2433-2443. [PubMed: 24373018]

14. Koster M, van de Vossenberg J, Leong J, Weisbeek PJ. Identification and characterization of the pupB gene encoding an inducible ferric-pseudobactin receptor of Pseudomonas putida WCS358. Molecular Microbiology. 1993; 8:591-601. [PubMed: 8392140]

15. Koster M, van Klompenburg W, Bitter W, Leong J, Weisbeek P. Role for the outer membrane ferric siderophore receptor PupB in signal transduction across the bacterial cell envelope. Embo J. 1994; 13:2805-2813. [PubMed: 8026465] 
16. Van Hove B, Staudenmaier H, Braun V. Novel two-component transmembrane transcription control: regulation of iron dicitrate transport in Escherichia coli K-12. J Bacteriol. 1990; 172:6749-6758. [PubMed: 2254251]

17. Enz S, Mahren S, Stroeher UH, Braun V. Surface Signaling in Ferric Citrate Transport Gene Induction: Interaction of the FecA, FecR, and FecI Regulatory Proteins. The Journal of Bacteriology. 2000; 182:637-646. [PubMed: 10633096]

18. Harle C, Kim I, Angerer A, Braun V. Signal transfer through three compartments: transcription initiation of the Escherichia coli ferric citrate transport system from the cell surface. EMBO J. 1995; 14:1430-1438. [PubMed: 7729419]

19. Wirth C, Meyer-Klaucke W, Pattus F, Cobessi D. From the Periplasmic Signaling Domain to the Extracellular Face of an Outer Membrane Signal Transducer of Pseudomonas aeruginosa: Crystal Structure of the Ferric Pyoverdine Outer Membrane Receptor. Journal of Molecular Biology. 2007; 368:398-406. [PubMed: 17349657]

20. Edgar RJ, Xu X, Shirley M, Konings AF, Martin LW, Ackerley DF, Lamont IL. Interactions between an anti-sigma protein and two sigma factors that regulate the pyoverdine signaling pathway in Pseudomonas aeruginosa. BMC Microbiol. 2014; 14:287. [PubMed: 25433393]

21. Malki I, Simenel C, Wojtowicz H, de Amorim GC, Prochnicka-Chalufour A, Hoos S, Raynal B, England P, Chaffotte A, Delepierre M, Delepelaire P, Izadi-Pruneyre N. Interaction of a Partially Disordered Antisigma Factor with Its Partner, the Signaling Domain of the TonB-Dependent Transporter HasR. PloS one. 2014; 9

22. Rossi MS, Paquelin A, Ghigo JM, Wandersman C. Haemophore-mediated signal transduction across the bacterial cell envelope in Serratia marcescens: the inducer and the transported substrate are different molecules. Molecular microbiology. 2003; 48:1467-1480. [PubMed: 12791131]

23. Biville F, Cwerman H, Letoffe S, Rossi MS, Drouet V, Ghigo JM, Wandersman C. Haemophoremediated signalling in Serratia marcescens: a new mode of regulation for an extra cytoplasmic function (ECF) sigma factor involved in haem acquisition. Molecular microbiology. 2004; 53:1267-1277. [PubMed: 15306027]

24. Krieg S, Huche F, Diederichs K, Izadi-Pruneyre N, Lecroisey A, Wandersman C, Delepelaire P, Welte W. Heme uptake across the outer membrane as revealed by crystal structures of the receptor-hemophore complex. P Natl Acad Sci USA. 2009; 106:1045-1050.

25. Mettrick KA, Lamont IL. Different roles for anti-sigma factors in siderophore signalling pathways of Pseudomonas aeruginosa. Molecular microbiology. 2009; 74:1257-1271. [PubMed: 19889096]

26. Ochs M, Veitinger S, Kim I, Weiz D, Angerer A, Braun V. Regulation of citrate-dependent iron transport of Escherichia coli: FecR is required for transcription activation by Feel. Molecular Microbiology. 1995; 15:119-132. [PubMed: 7752886]

27. Draper RC, Martin LW, Beare PA, Lamont IL. Differential proteolysis of sigma regulators controls cell-surface signalling in Pseudomonas aeruginosa. Mol Microbiol. 2011; 82:1444-1453. [PubMed: 22040024]

28. Bastiaansen KC, van Ulsen P, Wijtmans M, Bitter W, Llamas MA. Self-cleavage of the Pseudomonas aeruginosa Cell-surface Signaling Anti-sigma Factor FoxR Occurs through an N-O Acyl Rearrangement. The Journal of biological chemistry. 2015; 290:12237-12246. [PubMed: 25809487]

29. Bastiaansen KC, Otero-Asman JR, Luirink J, Bitter W, Llamas MA. Processing of cell-surface signalling anti-sigma factors prior to signal recognition is a conserved autoproteolytic mechanism that produces two functional domains. Environmental microbiology. 2015

30. Campbell EA, Greenwell R, Anthony JR, Wang S, Lim L, Das K, Sofia HJ, Donohue TJ, Darst SA. A conserved structural module regulates transcriptional responses to diverse stress signals in bacteria. Mol Cell. 2007; 27:793-805. [PubMed: 17803943]

31. Greenwell R, Nam TW, Donohue TJ. Features of Rhodobacter sphaeroides ChrR required for stimuli to promote the dissociation of sigma(E)/ChrR complexes. Journal of Molecular Biology. 2011; 407:477-491. [PubMed: 21295582]

32. Maillard AP, Girard E, Ziani W, Petit-Hartlein I, Kahn R, Coves J. The crystal structure of the anti-sigma factor $\mathrm{CnrY}$ in complex with the sigma factor $\mathrm{CnrH}$ shows a new structural class of 
anti-sigma factors targeting extracytoplasmic function sigma factors. Journal of Molecular Biology. 2014; 426:2313-2327. [PubMed: 24727125]

33. Ades SE, Connolly LE, Alba BM, Gross CA. The Escherichia coli sigma(E)-dependent extracytoplasmic stress response is controlled by the regulated proteolysis of an anti-sigma factor. Genes Dev. 1999; 13:2449-2461. [PubMed: 10500101]

34. Campbell EA, Tupy JL, Gruber TM, Wang S, Sharp MM, Gross CA, Darst SA. Crystal structure of Escherichia coli sigmaE with the cytoplasmic domain of its anti-sigma RseA. Mol Cell. 2003; 11:1067-1078. [PubMed: 12718891]

35. Newman JD, Anthony JR, Donohue TJ. The importance of zinc-binding to the function of Rhodobacter sphaeroides ChrR as an anti-sigma factor. Journal of Molecular Biology. 2001; 313:485-499. [PubMed: 11676534]

36. Thakur KG, Praveena T, Gopal B. Structural and biochemical bases for the redox sensitivity of Mycobacterium tuberculosis RslA. Journal of Molecular Biology. 2010; 397:1199-1208. [PubMed: 20184899]

37. Shukla J, Gupta R, Thakur KG, Gokhale R, Gopal B. Structural basis for the redox sensitivity of the Mycobacterium tuberculosis SigK-RskA sigma-anti-sigma complex. Acta crystallographica Section D, Biological crystallography. 2014; 70:1026-1036.

38. Sheffield P, Garrard S, Derewenda Z. Overcoming expression and purification problems of RhoGDI using a family of "Parallel" expression vectors. Protein Expression and Purification. 1999; 15:34-39. [PubMed: 10024467]

39. Ausubel, FM.; Brent, R.; Kingston, RE.; Moore, DD.; Seidman, JG.; Smith, HA.; Struhl, K. Current protocols in molecular biology. Greene Publishing Associates; New York: 1991.

40. Van Duyne GD, Standaert RF, Karplus PA, Schreiber SL, Clardy J. Atomic structures of the human immunophilin FKBP-12 complexes with FK506 and rapamycin. Journal of Molecular Biology. 1993; 229:105-124. [PubMed: 7678431]

41. Doublie S. Preparation of selenomethionyl proteins for phase determination. Methods Enzymol. 1997; 276:523-530. [PubMed: 9048379]

42. Kabsch W. Xds. Acta Cryst. 2010; D66:125-132.

43. Terwilliger TC, Adams PD, Read RJ, McCoy AJ, Moriarty NW, Grosse-Kunstleve RW, Afonine PV, Zwart PH, Hung LW. Decision-making in structure solution using Bayesian estimates of map quality: the PHENIX AutoSol wizard. Acta Crystallographa. 2009; D65:582-601.

44. Terwilliger TC, Grosse-Kunstleve RW, Afonine PV, Moriarty NW, Zwart PH, Hung LW, Read RJ, Adams PD. Iterative model building, structure refinement and density modification with the PHENIX AutoBuild wizard. Acta Crystallographa. 2008; D64:61-69.

45. Adams PD, Afonine PV, Bunkoczi G, Chen VB, Davis IW, Echols N, Headd JJ, Hung LW, Kapral GJ, Grosse-Kunstleve RW. PHENIX: a comprehensive Python-based system for macromolecular structure solution. Acta Cryst. 2010; D66:213-221.

46. Emsley P, Cowtan K. Coot: model-building tools for molecular graphics. Acta Cryst. 2004; D60:2126-2132.

47. Rossmann MG, Arnold E. International Tables for Crystallography Volume F: Crystallography of biological macromolecules. IUCr. 2001

48. McCoy AJ, Grosse-Kunstleve RW, Adams PD, Winn MD, Storoni LC, Read RJ. Phaser crystallographic software. Journal of Applied Crystallography. 2007; 40:658-674. [PubMed: 19461840]

49. Painter J, Merritt EA. Optimal description of a protein structure in terms of multiple groups undergoing TLS motion. Acta crystallographica Section D, Biological crystallography. 2006; 62:439-450.

50. Krissinel E, Henrick K. Inference of macromolecular assemblies from crystalline state. J Mol Biol. 2007; 372:774-797. [PubMed: 17681537]

51. Petoukhov MV, Franke D, Shkumatov AV, Tria G, Kikhney AG, Gajda M, Gorba C, Mertens HD, Konarev PV, Svergun DI. New developments in the program package for small-angle scattering data analysis. Journal of Applied Crystallography. 2012; 45:342-350. [PubMed: 25484842] 
52. Konarev PV, Volkov VV, Sokolova AV, Koch MHJ, Svergun DI. PRIMUS: a Windows PC-based system for small-angle scattering data analysis. Journal of Applied Crystallography. 2003; 36:1277-1282.

53. Semenyuk AV, Svergun DI. Gnom - a Program Package for Small-Angle Scattering DataProcessing. Journal of Applied Crystallography. 1991; 24:537-540.

54. Svergun DI. Determination of the Regularization Parameter in Indirect-Transform Methods Using Perceptual Criteria. Journal of Applied Crystallography. 1992; 25:495-503.

55. Franke D, Svergun DI. DAMMIF, a program for rapid ab-initio shape determination in small-angle scattering. Journal of Applied Crystallography. 2009; 42:342-346.

56. Volkov VV, Svergun DI. Uniqueness of ab initio shape determination in small-angle scattering. Journal of Applied Crystallography. 2003; 36:860-864.

57. Wriggers W, Milligan RA, McCammon JA. Situs: A package for docking crystal structures into low-resolution maps from electron microscopy. Journal of Structural Biology. 1999; 125:185-195. [PubMed: 10222274]

58. Wriggers W, Chacon P. Using Situs for the registration of protein structures with low-resolution bead models from X-ray solution scattering. Journal of Applied Crystallography. 2001; 34:773776.

59. Pettersen EF, Goddard TD, Huang CC, Couch GS, Greenblatt DM, Meng EC, Ferrin TE. UCSF chimera - A visualization system for exploratory research and analysis. Journal of Computational Chemistry. 2004; 25:1605-1612. [PubMed: 15264254]

60. Svergun D, Barberato C, Koch MHJ. CRYSOL - A program to evaluate x-ray solution scattering of biological macromolecules from atomic coordinates. Journal of Applied Crystallography. 1995; 28:768-773.

61. Fischer H, Neto MD, Napolitano HB, Polikarpov I, Craievich AF. Determination of the molecular weight of proteins in solution from a single small-angle $\mathrm{X}$-ray scattering measurement on a relative scale. Journal of Applied Crystallography. 2010; 43:101-109.

62. Schneidman-Duhovny D, Hammel M, Tainer JA, Sali A. Accurate SAXS Profile Computation and its Assessment by Contrast Variation Experiments. Biophysical journal. 2013; 105:962-974. [PubMed: 23972848]

63. Schneidman-Duhovny D, Hammel M, Sali A. FoXS: a web server for rapid computation and fitting of SAXS profiles. Nucleic Acids Research. 2010; 38:W540-W544. [PubMed: 20507903]

64. Tria G, Mertens HD, Kachala M, Svergun DI. Advanced ensemble modelling of flexible macromolecules using X-ray solution scattering. IUCrJ. 2015; 2:207-217.

65. Bernado P, Mylonas E, Petoukhov MV, Blackledge M, Svergun DI. Structural characterization of flexible proteins using small-angle X-ray scattering. J Am Chem Soc. 2007; 129:5656-5664. [PubMed: 17411046]

66. Zhao H, Berger AJ, Brown PH, Kumar J, Balbo A, May CA, Casillas E Jr, Laue TM, Patterson GH, Mayer ML, Schuck P. Analysis of high-affinity assembly for AMPA receptor amino-terminal domains. J Gen Physiol. 2013; 141:747-749. [PubMed: 23855058]

67. Brown PH, Balbo A, Schuck P. Characterizing protein-protein interactions by sedimentation velocity analytical ultracentrifugation. Curr Protoc Immunol Chapter. 2008; 18(Unit 18):15.

68. Zhao H, Brown PH, Schuck P. On the distribution of protein refractive index increments. Biophysical journal. 2011; 100:2309-2317. [PubMed: 21539801]

69. Schuck P. On the analysis of protein self-association by sedimentation velocity analytical ultracentrifugation. Analytical biochemistry. 2003; 320:104-124. [PubMed: 12895474]

70. Zhao H, Schuck P. Global multi-method analysis of affinities and cooperativity in complex systems of macromolecular interactions. Anal Chem. 2012; 84:9513-9519. [PubMed: 23020071]

71. Johnson ML. Why, When, and How Biochemists Should Use Least-Squares. Analytical biochemistry. 1992; 206:215-225. [PubMed: 1443589]

72. Perez-Iratxeta C, Andrade-Navarro MA. K2D2: estimation of protein secondary structure from circular dichroism spectra. BMC Struct Biol. 2008; 8

73. Sreerama N, Venyaminov SY, Woody RW. Analysis of protein circular dichroism spectra based on the tertiary structure classification. Analytical biochemistry. 2001; 299:271-274. [PubMed: 11730356] 
74. Angerer A, Enz S, Ochs M, Braun V. Transcriptional regulation of ferric citrate transport in Escherichia coli K-12. Fecl belongs to a new subfamily of sigma 70-type factors that respond to extracytoplasmic stimuli. Molecular microbiology. 1995; 18:163-174. [PubMed: 8596456]

75. Lambert LJ, Wei Y, Schirf V, Demeler B, Werner MH. T4 AsiA blocks DNA recognition by remodeling sigma70 region 4. EMBO J. 2004; 23:2952-2962. [PubMed: 15257291] 

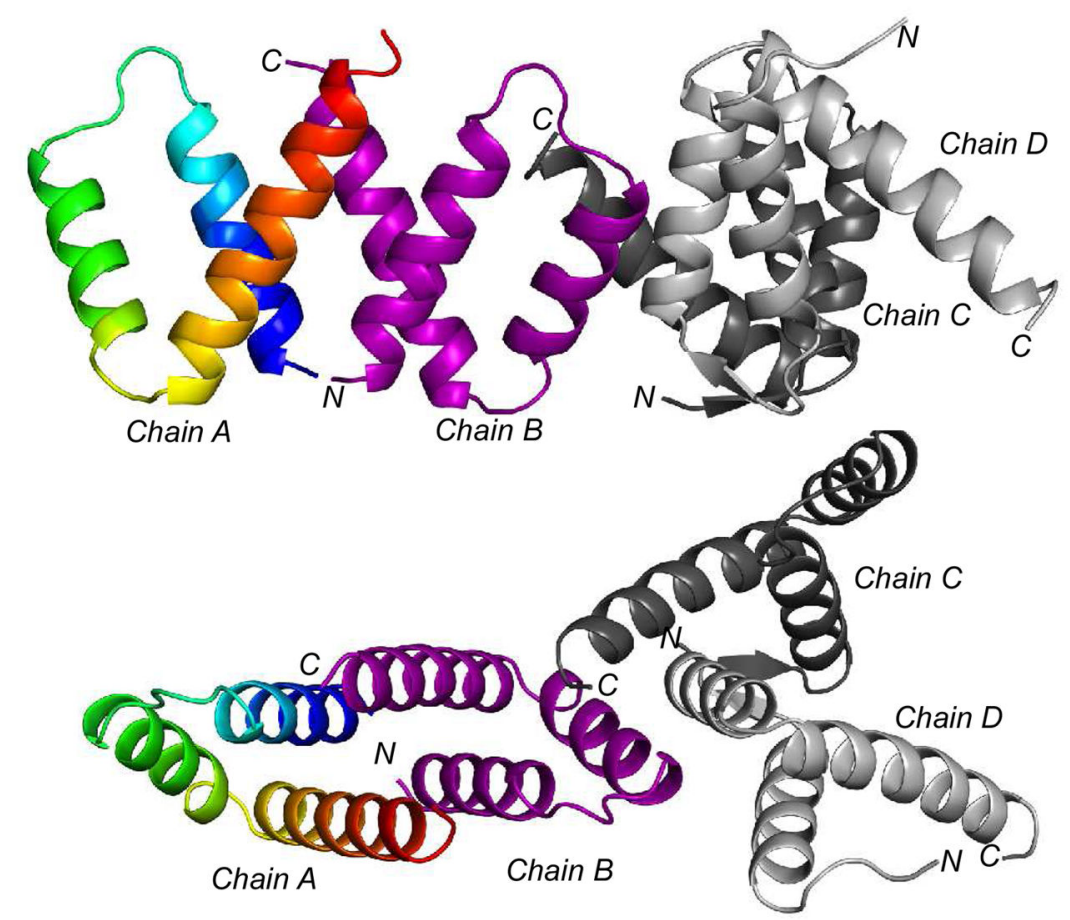

Figure 1.

The asymmetric unit of the PupR-ASD crystal. The four PupR-ASD monomers in the asymmetric unit are displayed in ribbon. Monomer A is rendered in rainbow colors with blue at the $\mathrm{N}$-terminus and red at the $\mathrm{C}$-terminus with Monomer B in purple. Monomers $\mathrm{C}$ and $\mathrm{D}$ are displayed in shades of gray. The symmetric dimer comprises chains $\mathrm{A}$ and $\mathrm{B}$; chains $\mathrm{C}$ and $\mathrm{D}$ are related by non-crystallographic symmetry to each other and to chains A and B. This and all molecular figures were made with PyMOL. 


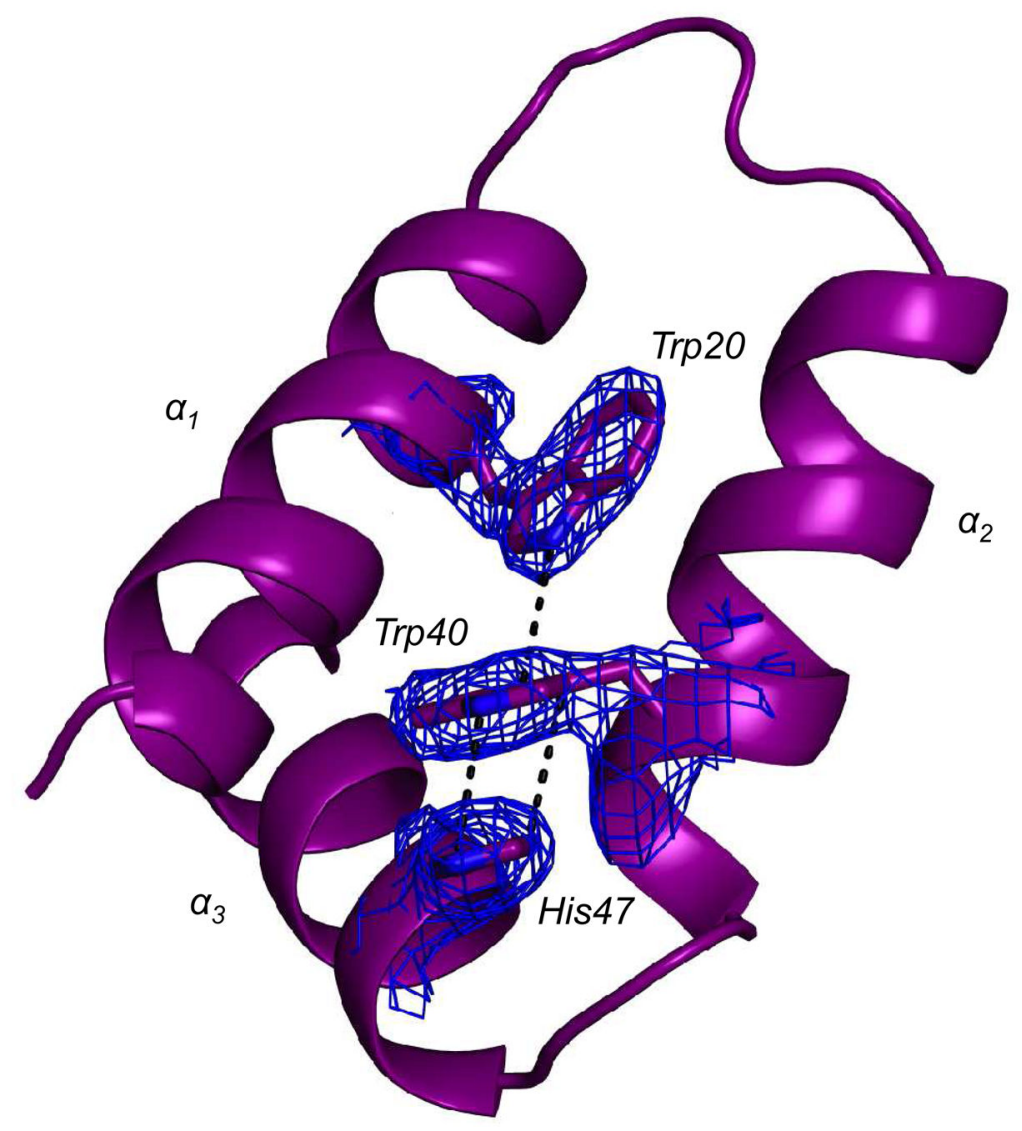

Figure 2.

Electron density (blue mesh) for the conserved aromatic residues involved in stabilizing the PupR-ASD core. Helixes 1-3 of the PupR-ASD monomer form the core of the ASD, with off-centered parallel $\pi-\pi$ stacking of His47 and Trp40, which is further stabilized by Trp 20 . The ASD backbone is displayed as a purple ribbon, with aromatic residues in stick, colorcoded by atom type: $\mathrm{N}$, blue; $\mathrm{O}$, red; $\mathrm{S}$, yellow and $\mathrm{C}$, magenta. 

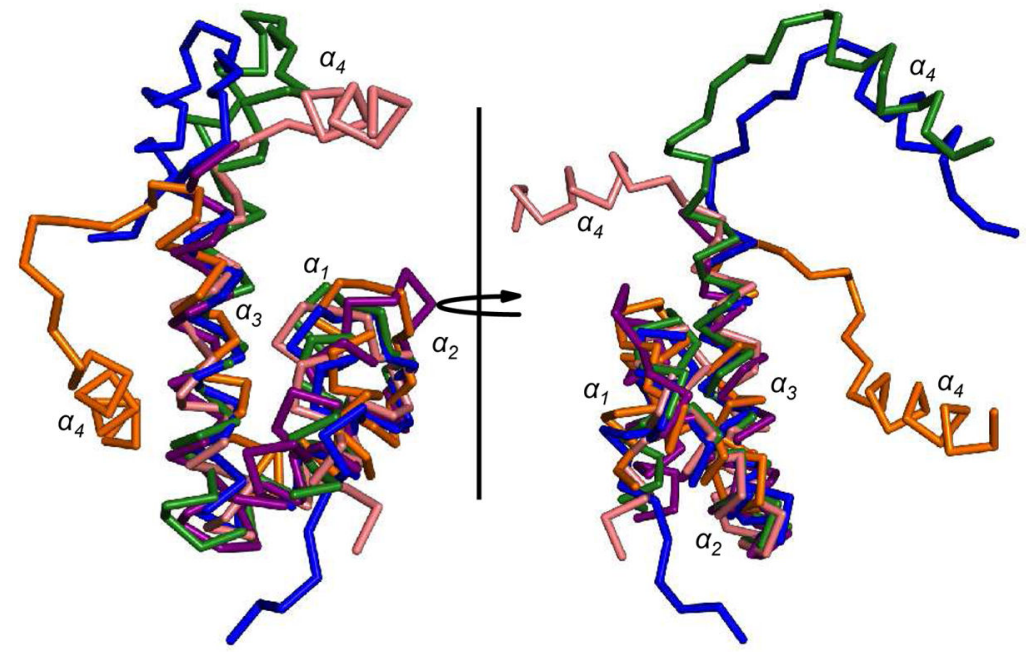

Figure 3.

Known ASD structures contain a conserved core helical bundle. Helices 1-3 of $R$. sphaeroides ChrR (blue; PDB entry 2Z2S; Campbell et al., 2007), M. tuberculosis RskA (green; PDB entry 4NQW; Shukla et al., 2014), M. tuberculosis RslA (salmon; PDB entry 3HUG; Thakur et al., 2010), and E. coli RseA (orange; PDB entry 1OR7; Campbell et al., 2003) were superimposed upon the PupR-ASD (purple). 

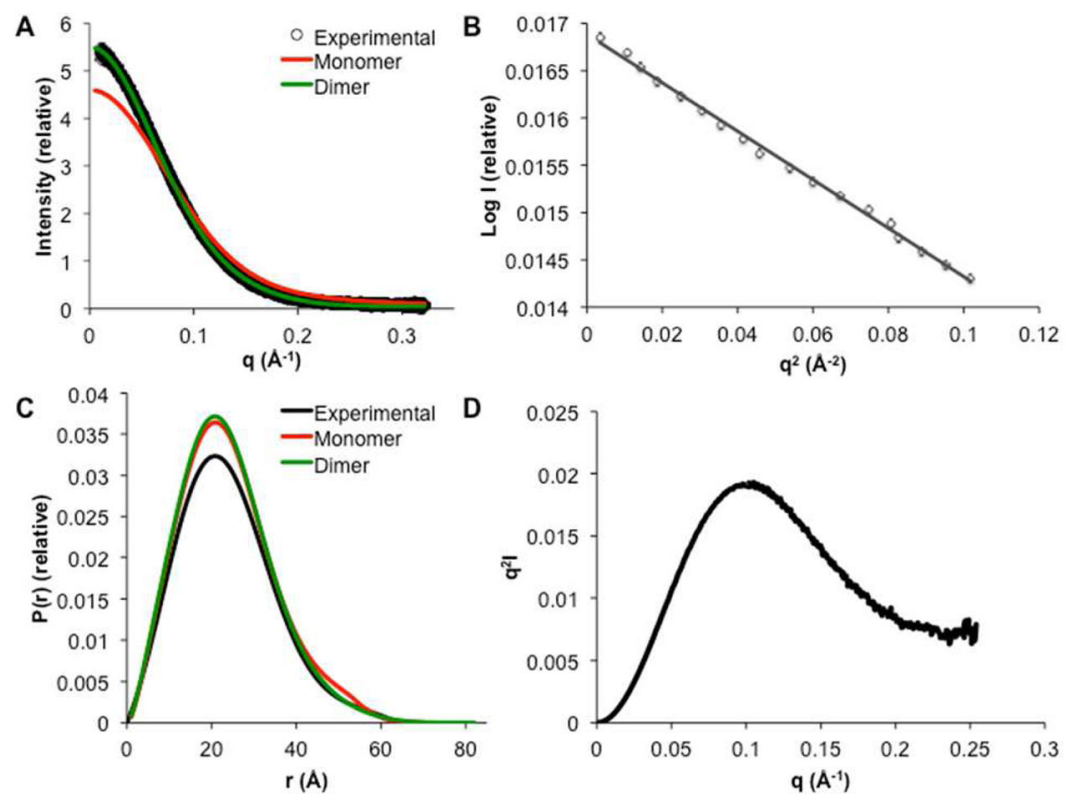

Figure 4.

SEC-SAXS analysis of PupR-ASD. A) The experimental scattering profile of the PupRASD (black) and the theoretical scattering profiles of the PupR-ASD dimer (green) and monomer (red) calculated from the X-ray crystal structure. B) The Guinier plot of the low $q$ region of the X-ray scattering data. C) The distance distribution $\mathrm{P}(\mathrm{r})$ plot for the experimental data (black) and the theoretical curves calculated for the PupR-ASD monomer (red) and dimer (dimer). D) The Kratky plot calculated from the experimental scattering profile. 


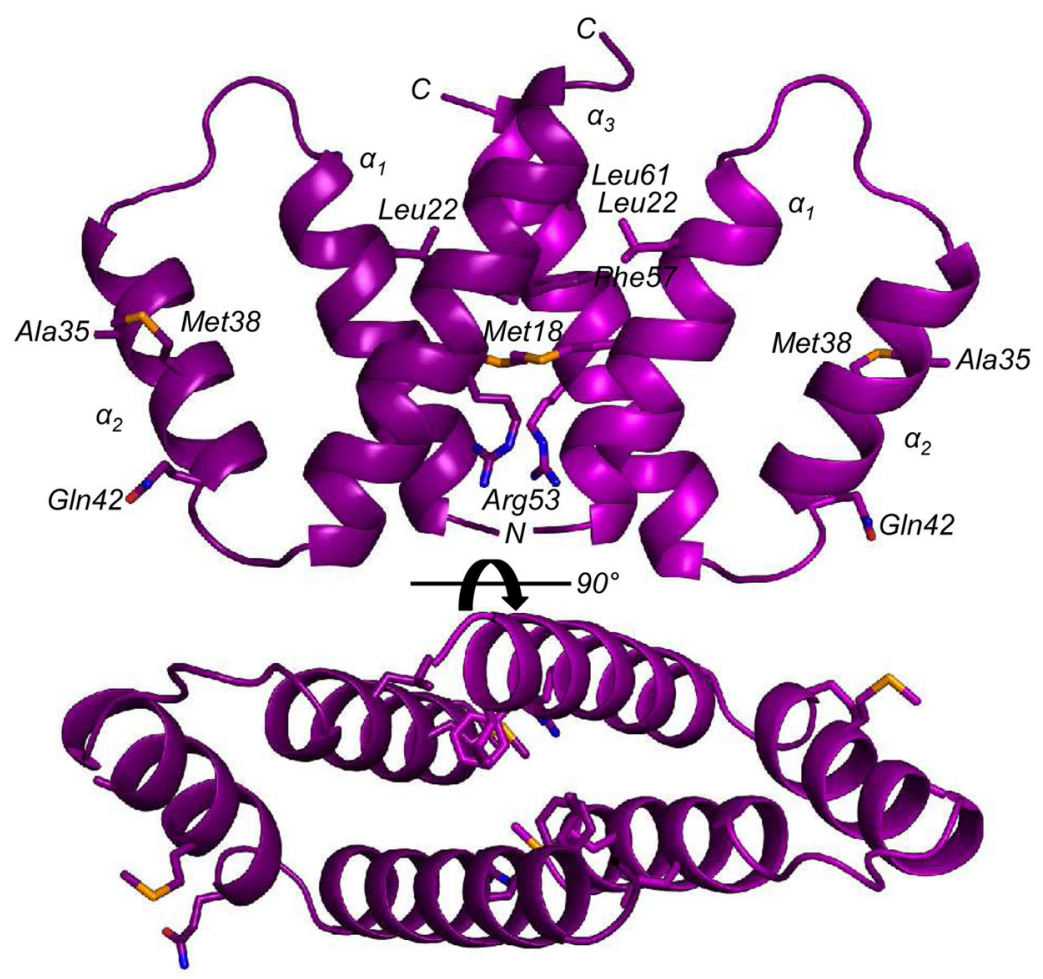

Figure 5.

The PupR-ASD symmetric dimer interface. Residues selected for mutation to aspartic acid are shown in stick and colored by atom type as in Figure 2. 


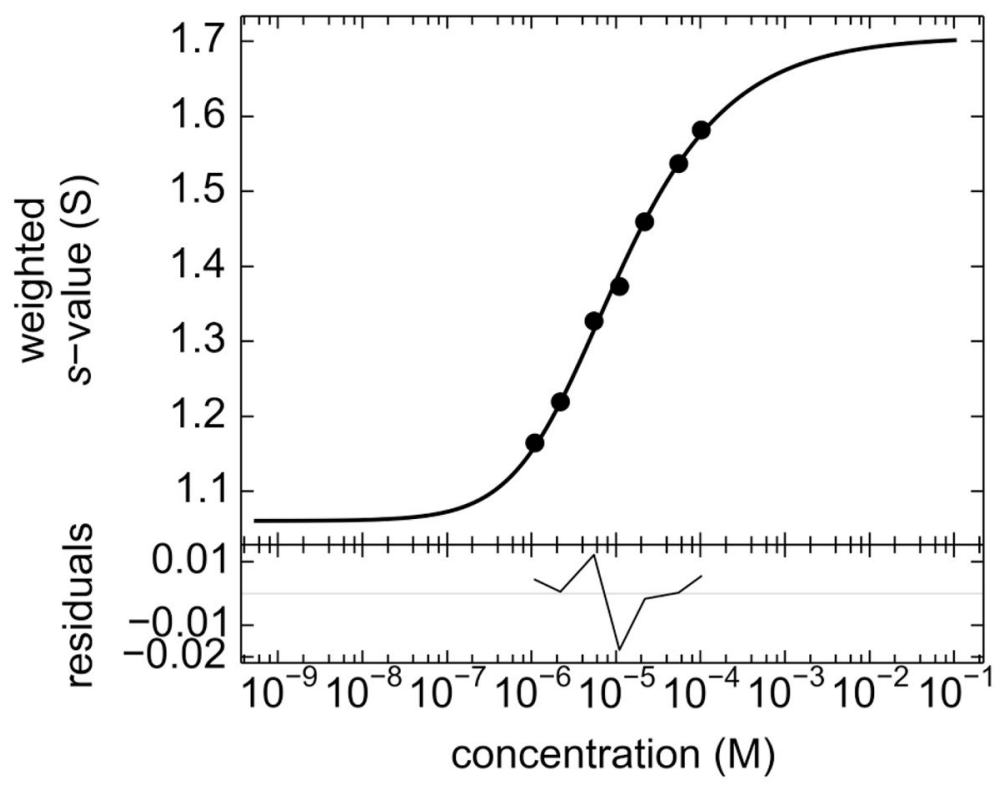

Figure 6.

$s_{w}$ isotherm for WT PupR-ASD created by integration of the $c(s)$ peaks in the distribution between 0 and $4 \mathrm{~S}$ for the data recorded at $20^{\circ} \mathrm{C}$, with error estimates calculated using SEDFIT. 


\section{Table 1}

X-ray Data Collection, Phasing, and Refinement Statistics ${ }^{a}$

\begin{tabular}{|c|c|c|}
\hline & Native & SeMet derivative \\
\hline \multicolumn{3}{|l|}{ Data Collection } \\
\hline Beamline & APS 24ID-C & APS 24ID-E \\
\hline \multicolumn{3}{|l|}{ Wavelength $(\AA)$} \\
\hline Space group & $P 2_{1}$ & $P 2_{1}$ \\
\hline Unit-cell parameters $\left(\AA{ }^{\circ}\right)$ & $33.5,134.6,34.990,113.7,90$ & $33.5,134.7,34.990,113.8,90$ \\
\hline Molecules in asymmetric unit & 4 & 4 \\
\hline Resolution range $(\AA)$ & $44.87-2.02(2.13-2.02)$ & $134.7-2.17(2.29-2.17)$ \\
\hline Total observations & $62331(9246)$ & $99992(5749)$ \\
\hline Unique observations & $18320(2685)$ & $14546(1796)$ \\
\hline Multiplicity & $3.4(3.4)$ & $6.9(3.2)$ \\
\hline Completeness (\%) & $98.8(99.4)$ & $97.2(82.0)$ \\
\hline $\mathrm{R}_{\text {merge }}(\%)$ & $4.5(44.2)$ & $11.3(71.1)$ \\
\hline $\mathrm{R}_{\text {merge }}($ anom) $(\%)$ & & $6.6(59.4)$ \\
\hline Average I/ $/ \mathrm{I}$ & $13.3(2.4)$ & $18.2(1.7)$ \\
\hline Data-processing program & RAPD & RAPD \\
\hline \multicolumn{3}{|l|}{ Refinement } \\
\hline Refinement program & PHENIX & PHENIX \\
\hline Resolution range $(\AA)$ & $33.66-2.02(2.09-2.02)$ & $67.35-2.17(2.25-2.17)$ \\
\hline $\mathrm{R}_{\text {work }}(\%)$ & 21.6 & 17.8 \\
\hline $\mathrm{R}_{\text {free }}(\%)$ & 25.7 & 21.7 \\
\hline \multicolumn{3}{|l|}{ RMSD stereochemistry } \\
\hline Bond lengths $(\AA)$ & 0.010 & 0.018 \\
\hline Bond angles $\left({ }^{\circ}\right)$ & 1.19 & 1.51 \\
\hline No. of atoms & 2023 & 2002 \\
\hline PupR-ASD & 1920 & 1906 \\
\hline Waters & 103 & 96 \\
\hline \multicolumn{3}{|l|}{ Average $B\left(\AA^{2}\right)$} \\
\hline PupR-ASD & 51.9 & 55.0 \\
\hline Waters & 49.7 & 45.6 \\
\hline \multicolumn{3}{|l|}{ Ramachandran plot (\%) } \\
\hline Preferred & 99 & 99 \\
\hline Allowed & 1 & 1 \\
\hline Outliers & 0 & 0 \\
\hline PDB code & $5 \mathrm{COS}$ & $5 \mathrm{CAM}$ \\
\hline
\end{tabular}

${ }^{a}$ Values in parentheses pertain to the highest resolution shell. 
Table 2

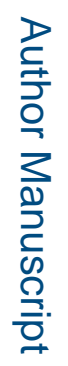

Melting temperatures determined by CD and estimated $K_{d}$ 's determined by SV-AUC for PupR-ASD constructs.

\begin{tabular}{|l|c|c|c|}
\hline Protein & $\mathbf{T}_{\mathbf{m}}\left({ }^{\circ} \mathbf{C}\right)$ & $\boldsymbol{K}_{\boldsymbol{d}}(\boldsymbol{\mu M})$ & AUC Concentrations $(\boldsymbol{\mu M})$ \\
\hline WT & 53.3 & 10.2 & $1.1-102$ \\
\hline M18D & 37.3 & 226 & $5.0,63.1$ \\
\hline L22D & 43.3 & $>200$ & 9.45 \\
\hline A35D & 44.0 & 13.5 & $6.36,50.0$ \\
\hline M38D & 44.7 & 11.6 & $4.39,51.21$ \\
\hline Q42D & 43.7 & 18.2 & $6.84,45.67$ \\
\hline R53D & 47.7 & 562 & $5.27,41.92$ \\
\hline F57D & 48.4 & $>200$ & 12.3 \\
\hline L61D & 48.8 & $>500$ & 103.7 \\
\hline
\end{tabular}

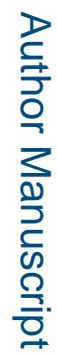

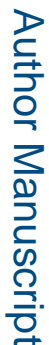

